\title{
THE BERNSTEIN-VON MISES THEOREM AND SPECTRAL ASYMPTOTICS OF BAYES ESTIMATORS FOR PARABOLIC SPDES
}

\author{
J. P. N. BISHWAL \\ (Received 30 August 2000; revised 28 February 2001) \\ Communicated by V. T. Stefanov
}

\begin{abstract}
The Bernstein-von Mises theorem, concerning the convergence of suitably normalized and centred posterior density to normal density, is proved for a certain class of linearly parametrized parabolic stochastic partial differential equations (SPDEs) as the number of Fourier coefficients in the expansion of the solution increases to infinity. As a consequence, the Bayes estimators of the drift parameter, for smooth loss functions and priors, are shown to be strongly consistent, asymptotically normal and locally asymptotically minimax (in the Hajek-Le Cam sense), and asymptotically equivalent to the maximum likelihood estimator as the number of Fourier coefficients become large. Unlike in the classical finite dimensional SDEs, here the total observation time and the intensity of noise remain fixed.
\end{abstract}

2000 Mathematics subject classification: primary $60 \mathrm{H} 15,62 \mathrm{M} 05,62 \mathrm{~F} 12,62 \mathrm{~F} 15$.

Keywords and phrases: stochastic partial differential equations, diffusion field, Bernstein-von Mises theorem, Bayes estimator, consistency, asymptotic normality, local asymptotic minimaxity, asymptotic equivalence, spectral theory.

\section{Introduction}

Recently infinite dimensional stochastic differential equations (SDEs), like the stochastic partial differential equations (SPDEs) are being paid a lot of attention in view of their modeling applications in neurophysiology, turbulence, oceanography and finance, see Itô [18], Walsh [34] and Kallianpur and Xiong [19], Holden et al. [12] and Carmona and Rozovskii [10]. In view of this it becomes necessary to estimate the unknown parameters in SPDEs.

Various methods of estimation in finite dimensional SDEs has been extensively studied during the last three decades as the observation time tends to infinity (see,

(C) 2002 Australian Mathematical Society $1446-7887 / 2002 \$ A 2.00+0.00$ 
Liptser and Shiryayev [25], Basawa and Prakasa Rao [1], Prakasa Rao [33] and Kutoyants [24]) or as the intensity of noise tends to zero (see, Ibragimov and Has'minskii [17], Kutoyants $[22,23])$. On the other hand, this problem for infinite dimensional SDEs is young. Loges [26] initiated the study of parameter estimation in such models. When the length of the observation time becomes large, he obtained consistency and asymptotic normality of the maximum likelihood estimator (MLE) of a real valued drift parameter in a Hilbert space valued SDE. Koski and Loges [21] extended the work of Loges [26] to minimum contrast estimators. Koski and Loges [20] applied the work to a stochastic heat flow problem.

Huebner, Khasminskii and Rozovskii [14] introduced spectral method to study consistency, asymptotic normality and asymptotic efficiency of MLE of a parameter in the drift coefficient of an SPDE. This approach allows one to obtain asymptotics of estimators under conditions which guarantee the singularity of the measures generated by the corresponding diffusion field for different parameters. Unlike in the finite dimensional cases, where the total observation time was assumed to be long $(T \rightarrow \infty)$ or intensity of the noise was assumed to be small $(\epsilon \rightarrow 0)$, here both are kept fixed. Here the asymptotics are obtained when the number of Fourier coefficients $(n)$ of the solution of SPDE becomes large.

The spectral asymptotics for MLE was extended by Huebner and Rozovskii [15] to more general SPDEs where the partial differential operators commute and satisfy some order conditions. Piterberg and Rozovskii [29] studied the properties MLE of a parameter in SPDE which are used to model the upper ocean variability in physical oceanography. Piterbarg and Rozovskii (1996) studied the properties of MLE based on discrete observations of the corresponding diffusion field. Huebner [13] extended the problem to the ML estimation of multidimensional parameter. Lototsky and Rozovskii [27] studied the same problem without the commutativity condition.

The Bernstein-von Mises theorem, concerning the convergence of suitably normalised and centered posterior distribution to normal distribution, plays a fundamental role in asymptotic Bayesian inference, see Le Cam and Yang [9]. In the i.i.d. case, the theorem was first proved by Le Cam [8]. Since then the theorem has been extended to many depended cases. Borwanker et al. [6] obtained the theorem for discrete time Markov processes. For the linear homogeneous diffusion processes, the Bernsteinvon Mises theorem was proved by Prakasa Rao [31]. Prakasa Rao [32] extended the theorem to a two parameter diffusion field. Bose [7] extended the theorem to the homogeneous nonlinear diffusions and Mishra [28] to the nonhomogeneous diffusions. As a further refinement in Bernstein-von Mises theorem, Bishwal [3] obtained sharp rates of convergence to normality of the posterior distribution and the Bayes estimators.

All these above works on Bernstein-von Mises theorem are concerned with finite dimensional SDEs. Recently Bishwal [2] proved the Bernstein-von Mises theorem 
and obtained asymptotic properties of regular Bayes estimator of the drift parameter in a Hilbert space valued SDE when the corresponding diffusion process is observed continuously over a time interval $[0, T]$. The asymptotics are studied as $T \rightarrow \infty$ under the condition of absolute continuity of measures generated by the process. Results are illustrated for the example of an SPDE. The situation is analogous to the finite dimensional SDEs, where the measures are absolutely continuous.

Our aim here is to use the spectral approach to study Bernstein-von Mises theorem and Bayes estimation in parabolic SPDE.

\section{Model and preliminaries}

Let $(\Omega, \mathscr{F}, P)$ be a complete probability space on which is defined the parabolic SPDE

$$
d u^{\theta}(t, x)=A^{\theta} u^{\theta}(t, x) d t+d W(t, x), \quad 0 \leq t \leq T, x \in G
$$

with Dirichlet boundary conditions

$$
\begin{aligned}
& u(0, x)=u_{0}(x), \\
& \left.D^{\gamma} u(t, x)\right|_{\partial G}=0 \text { for all indices } \gamma \text { with }|\gamma| \leq m-1,
\end{aligned}
$$

where $A^{\theta}=\theta A_{1}+A_{0}, A_{1}$ and $A_{0}$ are partial differential operators of orders $m_{1}$ and $m_{2}$ respectively, $A^{\theta}$ has order $2 m=\max \left(m_{1}, m_{0}\right), W(t, x)$ is a cylindrical Brownian motion in $L^{2}([0, T] \times G)$, where $G$ is a bounded domain in $\mathbb{R}^{d}$ and $u_{0} \in L_{2}(G)$. Here $\theta \in \Theta \subseteq \mathbb{R}$ is the unknown parameter to be estimated on the basis of the observations of the field $u^{\theta}(t, x), t \in[0, T], x \in G$. Let $\theta_{0}$ be the true value of the unknown parameter.

Here $u^{\theta}(t, x)$ is the observation at time $t$ at point $x$. In practice, it is impossible to observe the field $u^{\theta}(t, x)$ at all points $t$ and $x$. Hence, it is assumed that only finite dimensional projections $u^{n}:=u^{n, \theta}=\left(u_{1}^{\theta}(t), \ldots, u_{n}^{\theta}(t)\right), t \in[0, T]$ of the solution of (2.1) are available. In other words, we observe the first $n$ highest nodes in the Fourier expansion

$$
u^{\theta}(t, x)=\sum_{i=1}^{\infty} u_{i}^{\theta}(t) \phi_{i}(x)
$$

corresponding to some orthogonal basis $\left\{\phi_{i}(x)\right\}_{i=1}^{\infty}$. We consider observation continuous in time $t \in[0, T]$. Note that $u_{i}^{\theta}(t), i \geq 1$ are independent one dimensional Ornstein-Uhlenbeck processes (see Huebner and Rozovskii [15]). Since here the basic set up is the same as in [15], for different terminology the reader is referred to [15].

The following conditions are assumed: 
(H1) $m_{1} \geq m-d / 2$.

(H2) The operators $A_{1}$ and $A_{0}$ are formally self-adjoint, that is, for $i=0,1$,

$$
\int_{G} A_{i} u v d x=\int_{G} u A_{i} v d x \text { for all } u, v \in C_{0}^{\infty}(G) \text {. }
$$

(H3) There is a compact neighbourhood $\Theta$ of $\theta_{0}$ so that $\left\{A^{\theta}, \theta \in \Theta\right\}$ is a family of uniformly strongly elliptic operators.

(H4) There exists a complete orthonormal system $\left\{h_{i}\right\}_{i=1}^{\infty}$ in $L_{2}(G)$ such that for every $i=1,2, \ldots, h_{i} \in W_{0}^{m, 2}(G) \cap C^{\infty}(\bar{G})$ and

$$
\Lambda_{\theta} h_{i}=\lambda_{i}(\theta) h_{i} \quad \text { and } \quad \mathscr{L}_{\theta} h_{i}=\mu_{i}(\theta) h_{i} \quad \text { for all } \theta \in \Theta,
$$

where $\mathscr{L}_{\theta}$ is a closed self adjoint extension of $A^{\theta}, \Lambda_{\theta}:=\left(k(\theta) I-\mathscr{L}_{\theta}\right)^{1 / 2 m}, k(\theta)$ is a constant and the spectrum of the operator $\Lambda_{\theta}$ consists of eigenvalues $\left\{\lambda_{i}(\theta)\right\}_{i=1}^{\infty}$ of finite multiplicities and $\mu_{i}=-2 \lambda_{i}^{2 m}+k(\theta)$.

(H5) The operator $A_{1}$ is uniformly strongly elliptic and has the same system of eigen functions $\left\{h_{i}\right\}_{i=1}^{\infty}$ as $\mathscr{L}_{\theta}$.

For $\alpha>d / 2$, define the Hilbert space $H^{-\alpha}$ as in Huebner and Rozovskii [15]. Let $P_{\theta}^{T}$ be the measure generated by the solution $\left\{u^{\theta}(t, x), t \in[0, T], x \in G\right\}$ to the problem (2.1)-(2.3) on the space $\mathscr{C}\left([0, T] ; H^{-\alpha}\right)$ with the associated Borel $\sigma$-algebra $\mathscr{B}_{T}$. Note that, under (H1), for different $\theta$ the measures $P_{\theta}^{T}$ are singular.

Consider the projection of $H^{-\alpha}$ onto the subspace $\mathbb{R}^{n}$. Let $P_{\theta}^{T, n}$ be the measure generated by $u^{n, \theta}$ on $\mathscr{C}\left[(0, T] ; \mathbb{R}^{n}\right)$ with the associated Borel $\sigma$-algebra $\mathscr{B}_{T}^{n}$.

It is a classical fact (see Liptser and Shiryayev [25]) that for any $\theta \in \Theta$, the measures $P_{\theta}^{T, n}$ and $P_{\theta_{0}}^{T, n}$ are mutually absolutely continuous with Radon-Nikodym derivative (likelihood ratio) given by

$$
\begin{aligned}
Z_{n}^{\theta}(u):=\frac{d P_{\theta}^{T, n}}{d P_{\theta_{0}}^{T, n}}\left(u^{n}\right)= & \exp \left\{\left(\theta-\theta_{0}\right) \int_{0}^{T}\left(A_{1} u^{n}(s), d u^{n}(s)\right)_{0}\right. \\
& -\frac{1}{2}\left(\theta^{2}-\theta_{0}^{2}\right) \int_{0}^{T}\left\|A_{1} u^{n}(s)\right\|_{0}^{2} d s \\
& \left.-\left(\theta-\theta_{0}\right) \int_{0}^{T}\left(A_{1} u^{n}(s), A_{0} u^{n}(s)\right)_{0} d s\right\} .
\end{aligned}
$$

Maximizing $Z_{n}^{\theta}(u)$ with respect to $\theta$ provides the MLE given by

$$
\hat{\theta}^{n}:=\frac{\int_{0}^{T}\left(A_{1} u^{n}(s), d u^{n}(s)-A_{0} u^{n}(s) d s\right)_{0}}{\int_{0}^{T}\left\|A_{1} u^{n}(s)\right\|_{0}^{2} d s} .
$$

The Fisher information $I_{n}$ related to $d P_{\theta}^{n} / d P_{\theta_{0}}^{n}$ is given by

$$
I_{n}:=E_{\theta_{0}} \int_{0}^{T}\left\|A_{1} u^{n}(s)\right\|_{0}^{2} d s .
$$


Define

$$
\psi_{n}:= \begin{cases}(\zeta / 4 \beta) T n^{2 \beta}, & \text { if } m_{1}>m-d / 2 \\ (\zeta / 2) T \log n, & \text { if } m_{1}=m-d / 2\end{cases}
$$

where $\beta=\left(m_{1}-m\right) / d+1 / 2$,

$$
\zeta:=(2 \pi)^{2\left(m_{1}-m\right)} \frac{\left(\int_{P_{A} \theta_{(x, v)}<1} d x d v\right)^{2 m / d}}{\left(\int_{P_{A_{1}(x, v)}<1} d x d v\right)^{2 m_{1} / d}} .
$$

Note that $I_{n} / \psi_{n} \rightarrow 0$ as $n \rightarrow \infty$. Let $\omega$ be a real valued, non-negative loss function of polynomial majorant defined on $\mathbb{R}$, which is symmetric $\omega(0)=0$ and monotone on the positive real line.

Under the conditions (H1)-(H5), Huebner and Rozovskii [15] showed that $\hat{\theta}_{n}$ is strongly consistent, asymptotically normally distributed with normalization $\psi_{n}^{1 / 2}$ and asymptotically efficient with respect to the loss function $\omega$.

Suppose that $\Pi$ is a priori probability measure on $(\Theta, \mathscr{D})$, where $\mathscr{D}$ is the $\sigma$-algebra of Borel subsets of $\Theta$. Assume that $\Pi$ has a density $\pi(\cdot)$ with respect to the Lebesgue measure and the density is continuous and positive in an open neighbourhood of $\theta_{0}$.

The posterior density of $\theta$ given in $u^{n}$ is given by

$$
p\left(\theta \mid u^{n}\right):=\frac{Z_{n}^{\theta}(u) \pi(\theta)}{\int_{\Theta} Z_{n}^{\theta}(u) \pi(\theta) d \theta} .
$$

Let $\tau=\psi_{n}^{1 / 2}\left(\theta-\hat{\theta}^{n}\right)$. Then the posterior density of $\psi_{n}^{1 / 2}\left(\theta-\hat{\theta}^{n}\right)$ is given by

$$
p^{*}\left(\tau \mid u^{n}\right):=\psi_{n}^{-1 / 2} p\left(\hat{\theta}^{n}+\psi_{n}^{-1 / 2} \tau \mid u^{n}\right)
$$

Let

$$
\begin{aligned}
\nu_{n}(\tau) & :=\frac{d P_{\hat{\theta}^{n}+\psi_{1}^{-1 / 2} \tau}^{n} / d P_{\theta_{0}}^{n}}{d P_{\hat{\theta}^{n}}^{n} / d P_{\theta_{0}}^{n}}=\frac{d P_{\hat{\theta}^{n}+\psi_{n}^{-1 / 2} \tau}^{n}}{d P_{\hat{\theta}^{n}}^{n}}, \\
C_{n} & :=\int_{-\infty}^{\infty} v_{n}(\tau) \pi\left(\hat{\theta}^{n}+\psi_{n}^{-1 / 2} \tau\right) d \tau .
\end{aligned}
$$

Clearly, $p^{*}\left(\tau \mid u^{n}\right)=C_{n}^{-1} v_{n}(\tau) \pi\left(\hat{\theta}^{n}+\psi_{n}^{-1 / 2} \tau\right)$

\section{The Bernstein-von Mises theorem}

Let $K(\cdot)$ be a non-negative measurable function satisfying the following two conditions: 
(K1) There exists a number $\eta, 0<\eta<1$, for which

$$
\int_{-\infty}^{\infty} K(\tau) \exp \left\{-\tau^{2}(1-\eta) / 2\right\} d \tau<\infty
$$

(K2) For every $\epsilon>0$ and $\delta>0$

$$
\left.e^{-\epsilon \psi_{n}} \int_{|\tau|>\delta} K\left(\tau \psi_{n}^{1 / 2}\right) \pi\left(\hat{\theta}^{n}+\tau\right) d \tau \rightarrow 0 \text { a.s. [ } P_{\theta_{0}}\right] \text { as } n \rightarrow \infty
$$

We need the following lemma to prove the Bernstein-von Mises theorem.

LEMMA 3.1. Under the assumptions (H1)-(H5) and (K1)-(K2):

(i) There exists $\delta_{0}>0$ such that

$$
\left.\lim _{n \rightarrow \infty} \int_{|\tau| \leq \delta_{0} \psi_{n}^{1 / 2}} K(\tau)\left|v_{n}(\tau) \pi\left(\hat{\theta}^{n}+\psi_{n}^{-1 / 2} \tau\right)-\pi\left(\theta_{0}\right) e^{-\tau^{2} / 2}\right| d \tau=0 \text { a.s. [ } P_{\theta_{0}}\right]
$$

(ii) For every $\delta>0$,

$$
\left.\lim _{n \rightarrow \infty} \int_{|\tau| \geq \delta \psi_{n}^{1 / 2}} K(\tau)\left|v_{n}(\tau) \pi\left(\hat{\theta}^{n}+\psi_{n}^{-1 / 2} \tau\right)-\pi\left(\theta_{0}\right) e^{-\tau^{2} / 2}\right| d \tau=0 \text { a.s. [ } P_{\theta_{0}}\right]
$$

ProOF. From (2.4) and (2.7), it is easy to check that

$$
\log \nu_{n}(\tau)=-\frac{1}{2} \tau^{2} \psi_{n}^{-1} \int_{0}^{T}\left\|A_{1} u^{n}(s)\right\|_{0}^{2} d s
$$

Now (i) follows by an application of the dominated convergence theorem.

For every $\delta>0$, there exists $\epsilon>0$ depending on $\delta$ and $\beta$ such that

$$
\begin{aligned}
\int_{|\tau| \geq \delta \psi_{n}^{1 / 2}} K(\tau)\left|v_{n}(\tau) \pi\left(\hat{\theta}^{n}+\psi_{n}^{-1 / 2} \tau\right)-\pi\left(\theta_{0}\right) e^{-\tau^{2} / 2}\right| d \tau \\
\quad \leq \int_{|\tau| \geq \delta \psi_{n}^{1 / 2}} K(\tau) v_{n}(\tau) \pi\left(\hat{\theta}^{n}+\psi_{n}^{-1 / 2} \tau\right) d \tau+\int_{|\tau| \geq \delta \psi_{n}^{1 / 2}} \pi\left(\theta_{0}\right) e^{-\tau^{2} / 2} d \tau \\
\quad \leq e^{-\epsilon \psi_{n}} \int_{|\tau| \geq \delta \psi_{n}^{1 / 2}} K(\tau) \pi\left(\hat{\theta}^{n}+\psi_{n}^{-1 / 2} \tau\right) d \tau+\pi\left(\theta_{0}\right) \int_{|\tau| \geq \delta \psi_{n}^{1 / 2}} e^{-\tau^{2} / 2} d \tau \\
=: F_{n}+G_{n} .
\end{aligned}
$$

By condition (K2), it follows that $F_{n} \rightarrow 0$ a.s. $\left[P_{\theta_{0}}\right]$ as $n \rightarrow \infty$ for every $\delta>0$. Condition (K1) implies that $G_{n} \rightarrow 0$ as $n \rightarrow \infty$. This completes the proof of the lemma.

Now we are ready to prove the generalized version of the Bernstein-von Mises theorem for parabolic SPDEs. 
THEOREM 3.1. Under the assumptions $(\mathrm{H} 1)-(\mathrm{H} 5)$ and $(\mathrm{K} 1)-(\mathrm{K} 2)$, we have

$$
\lim _{n \rightarrow \infty} \int_{-\infty}^{\infty} K(\tau)\left|p^{*}\left(\tau \mid u^{n}\right)-(1 / 2 \pi)^{1 / 2} e^{-\tau^{2} / 2}\right| d \tau=0 \text { a.s. }\left[P_{\theta_{0}}\right]
$$

PROOF. From Lemma 3.1, we have

$$
\lim _{n \rightarrow \infty} \int_{-\infty}^{\infty} K(\tau)\left|\nu_{n}(\tau) \pi\left(\hat{\theta}^{n}+\psi_{n}^{-1 / 2} \tau\right)-\pi\left(\theta_{0}\right) e^{-\tau^{2} / 2}\right| d \tau=0 \text { a.s. }\left[P_{\theta_{0}}\right] .
$$

Putting $K(\tau)=1$ which trivially satisfies (K1) and (K2), we have

$$
C_{n}=\int_{-\infty}^{\infty} v_{n}(\tau) \pi\left(\hat{\theta}^{n}+\psi_{n}^{-1 / 2} \tau\right) d \tau \rightarrow \pi\left(\theta_{0}\right) \int_{-\infty}^{\infty} e^{-\tau^{2} / 2} d \tau \text { a.s. }\left[P_{\theta_{0}}\right]
$$

Therefore, by (3.1) and (3.2), we have

$$
\begin{aligned}
\int_{-\infty}^{\infty} K(\tau)\left|p^{*}\left(\tau \mid u^{n, \theta}\right)-(1 / 2 \pi)^{1 / 2} e^{-\tau^{2} / 2}\right| d \tau \\
\leq \int_{-\infty}^{\infty} K(\tau)\left|C_{n}^{-1} \nu_{n}(\tau) \pi\left(\hat{\theta}^{n}+\psi_{n}^{-1 / 2} \tau\right)-C_{n}^{-1} \pi\left(\theta_{0}\right) e^{-\tau^{2} / 2}\right| d \tau \\
\quad+\int_{-\infty}^{\infty} K(\tau)\left|C_{n}^{-1} \pi\left(\theta_{0}\right) e^{-\tau^{2} / 2}-(1 / 2 \pi)^{1 / 2} e^{-\tau^{2} / 2}\right| d \tau \stackrel{n \rightarrow \infty}{\longrightarrow} 0 \text { a.s. }\left[P_{\theta_{0}}\right]
\end{aligned}
$$

THEOREM 3.2. Suppose (H1)-(H5) and $\int_{-\infty}^{\infty}|\theta|^{r} \pi(\theta) d \theta<\infty$ for some nonnegative integer $r$ hold. Then

$$
\lim _{n \rightarrow \infty} \int_{-\infty}^{\infty}|\tau|^{r}\left|p^{*}\left(\tau \mid u^{n}\right)-(1 / 2 \pi)^{1 / 2} e^{-\tau^{2} / 2}\right| d \tau=0 \text { a.s. }\left[P_{\theta_{0}}\right]
$$

PROOF. For $r=0$, the verification of $(\mathrm{K} 1)$ and $(\mathrm{K} 2)$ is easy and the theorem follows from Theorem 3.1. Suppose $r \geq 1$. Let $K(\tau)=|\tau|^{r}, \delta>0$ and $\epsilon>0$. Using $|a+b|^{r} \leq 2^{r-1}\left(|a|^{r}+|b|^{r}\right)$, we have

$$
\begin{aligned}
e^{-\epsilon \psi_{n}} & \int_{|\tau|>\delta} K\left(\tau \psi_{n}^{1 / 2}\right) \pi\left(\hat{\theta}^{n}+\tau\right) d \tau \\
& \leq \psi_{n}^{r / 2} e^{-\epsilon \psi_{n}} \int_{\left|\tau-\hat{\theta}^{n}\right|>\delta} \pi(\tau)\left|\tau-\hat{\theta}^{n}\right|^{r} d \tau \\
& \leq 2^{r-1} \psi_{n}^{r / 2} e^{-\epsilon \psi_{n}}\left[\int_{\left|\tau-\hat{\theta}^{n}\right|>\delta} \pi(\tau)|\tau|^{r} d \tau+\int_{\left|\tau-\hat{\theta}^{n}\right|>\delta} \pi(\tau)\left|\hat{\theta}^{n}\right|^{r} d \tau\right] \\
& \leq 2^{r-1} \psi_{n}^{r / 2} e^{-\epsilon \psi_{n}}\left[\int_{-\infty}^{\infty} \pi(\tau)|\tau|^{r} d \tau+\left|\hat{\theta}^{n}\right|^{r}\right] \rightarrow 0 \text { a.s. }\left[P_{\theta_{0}}\right] \text { as } n \rightarrow \infty
\end{aligned}
$$

from the strong consistency of $\hat{\theta}^{n}$ and hypothesis of the theorem. Thus the theorem follows from Theorem 3.1. 
REMARK 3.1. For $r=0$ in Theorem 3.2, we have

$$
\lim _{n \rightarrow \infty} \int_{-\infty}^{\infty}\left|p^{*}\left(\tau \mid u^{n}\right)-(1 / 2 \pi)^{1 / 2} e^{-\tau^{2} / 2}\right| d \tau=0 \text { a.s. }\left[P_{\theta_{0}}\right] .
$$

This is the classical form of Bernstein-von Mises theorem for parabolic SPDEs in its simplest form.

As a special case of Theorem 3.2 , we obtain $E_{\theta_{0}}\left[\psi_{n}^{1 / 2}\left(\hat{\theta}^{n}-\theta_{0}\right)\right]^{r} \rightarrow E\left[\xi^{r}\right]$ as $n \rightarrow \infty$ where $\xi \sim \mathscr{N}(0,1)$.

\section{Bayes estimation}

As an application of Theorem 3.1, we obtain the asymptotic properties of a regular Bayes estimator of $\theta$. Suppose $l(\theta, \phi)$ is a loss function defined on $\Theta \times \Theta$. Assume that $l(\theta, \phi)=l(|\theta-\phi|) \geq 0$ and $l(\cdot)$ is non decreasing. Suppose that $J$ is a non-negative function on $\mathbb{N}$ and $K(\cdot)$ and $G(\cdot)$ are functions on $\mathbb{R}$ such that

(B1) $J(n) l\left(\tau \psi_{n}^{-1 / 2}\right) \leq G(\tau)$ for all $n$;

(B2) $J(n) l\left(\tau \psi_{n}^{-1 / 2}\right) \rightarrow K(\tau)$ as $n \rightarrow \infty$ uniformly on bounded subsets of $\mathbb{R}$;

(B3) $\int_{-\infty}^{\infty} K(\tau+s) e^{-\tau^{2} / 2} d \tau$ has a strict minimum at $s=0$;

(B4) $G(\cdot)$ satisfies (K1) and (K2).

Let $B_{n}(\phi)=\int_{\theta} l(\theta, \phi) p\left(\theta \mid u^{n}\right) d \theta$. A regular Bayes estimator $\tilde{\theta}^{n}$ based on $u^{n}$ is defined as $\tilde{\theta}^{n}:=\arg \inf _{\phi \in \Theta} B_{n}(\phi)$. Assume that such an estimator exists.

The following theorem shows that MLE and Bayes estimators are asymptotically equivalent as $n \rightarrow \infty$.

THEOREM 4.1. Assume that (H1)-(H5), (K1)-(K2) and (B1)-(B4) hold. Then we have

(i) $\psi_{n}^{1 / 2}\left(\tilde{\theta}^{n}-\hat{\theta}^{n}\right) \rightarrow 0$ a.s. $\left[P_{\theta_{0}}\right]$ as $n \rightarrow \infty$,

(ii) $\lim _{n \rightarrow \infty} J(n) B_{n}\left(\tilde{\theta}^{n}\right)=\lim _{n \rightarrow \infty} J(n) B_{n}\left(\hat{\theta}^{n}\right)=\left(\frac{1}{2 \pi}\right)^{1 / 2} \int_{-\infty}^{\infty} K(\tau) e^{-\tau^{2} / 2} d \tau$ a.s. [ $\left.P_{\theta_{0}}\right]$.

PROOF. The proof is analogous to Theorem 4.1 in Borwanker et al. [6]. We omit the details.

COROLLARY 4.2. Under the assumptions of Theorem 4.1, we have

(i) $\tilde{\theta}^{n} \rightarrow \theta_{0}$ a.s. $\left[P_{\theta_{0}}\right]$ as $n \rightarrow \infty$.

(ii) $\psi_{n}^{1 / 2}\left(\tilde{\theta}^{n}-\theta_{0}\right) \stackrel{\mathscr{L}}{\rightarrow} \mathscr{N}(0,1)$ as $n \rightarrow \infty$.

PROOF. (i) and (ii) follow easily by combining Theorem 4.1 and the strong consistency and asymptotic normality results of the MLE in Huebner and Rozovskii [15]. 
The following theorem shows that Bayes estimators are locally asymptotically minimax in the Hajek-Le Cam sense, that is, equality is achieved in the Hajek-Le Cam inequality.

THEOREM 4.3. Under the assumptions of Theorem 4.1, we have

$$
\lim _{\delta \rightarrow \infty} \lim _{n \rightarrow \infty} \sup _{\left|\theta-\theta_{0}\right|<\delta} E \omega\left(I_{n}^{1 / 2}\left(\tilde{\theta}^{n}-\theta_{0}\right)\right)=E \omega(\xi), \quad \mathscr{L}(\xi)=\mathscr{N}(0,1),
$$

where $\omega(\cdot)$ is a loss function as defined in theorem earlier.

PROOF. The theorem follows from Theorem III.2.1 in Ibragimov and Khasminskii [16] since here conditions (N1)-(N4) of the above mentioned theorem are satisfied using Lemma 3.1 - Lemma 3.3 and local asymptotic normality (LAN) property obtained in Huebner and Rozovskii [15].

\section{Example}

We illustrate the results of the previous sections through the following heat equation

$$
\begin{aligned}
& d u(t, x)-\theta \Delta u(t, x)=d W(t, x), \quad t \in[0, T], x \in(0,1) \\
& u(0, x)=u_{0}(x), \quad x \in(0,1) \\
& u(t, 0)=u(t, 1)=0, \quad t \in[0, T],
\end{aligned}
$$

where $u_{0} \in L_{2}(0,1), W(t, x)$ is a cylindrical Brownian motion in $L_{2}(0,1)$ and $\theta>0$.

Here $m_{1}=\operatorname{ord}(\Delta)=2, m_{0}=0, m=(1 / 2) \max \left(m_{1}, m_{0}\right)=1, d=1$. So $m-d / 2=1 / 2<m_{1}$. Thus (H1) is satisfied. By standard arguments, the operator $-\theta \Delta$ with zero boundary conditions extends to a self adjoint operator on $L_{2}(0,1)$ which we denote by $-\theta \Delta$. The domain $\mathscr{D}(-\theta \Delta)=W^{2,2}(0,1) \cap W_{0}^{1,2}(0,1)$. It is readily checked that $-\theta \Delta$ is positive, so we can take $k(\theta)=0$ and set $\Lambda=\sqrt{-\theta \Delta}$. It is a standard fact that $\mathscr{D}(\sqrt{-\theta \Delta})=W_{0}^{1,2}(0,1)$. Write $h_{i}:=\sqrt{2} \sin (i \pi x)$. Obviously the sequence $h_{i}, i=1,2, \ldots$, forms a complete orthonormal system in $L_{2}(0,1)$ and $\sqrt{-\theta \Delta} h_{i}=\lambda_{i}(\theta) h_{i}$ where $\lambda_{i}(\theta)=\sqrt{\theta} \pi i$. It is readily checked that for $s \in \mathbb{R}$, the norm

$$
\|u\|_{s}:=\left(2 \sum_{j=1}^{\infty} \theta^{s}(\pi i)^{2 s}\left|\int_{0}^{1} u(s) \sin (j \pi x) d x\right|^{2}\right)^{1 / 2}
$$

is equivalent to the norm of the Sobolev space $W_{0}^{s, 2}(0,1)$. Let us choose $\alpha=1$. Obviously the system $h_{i, \theta}^{-1}:=\lambda_{i} h_{i}=\sqrt{\theta} \pi i \sqrt{2} \sin (\pi i x), i=1,2, \ldots$, is an orthonormal basis in $H^{-1}$. Hence assumptions (H2)-(H5) are satisfied. 
Take squared error loss function $l(\theta, \phi)=|\theta-\phi|^{2}$. Now for this heat equation example all the results of Section 3 and Section 4 on posterior convergence and asymptotics of Bayes estimators, which are posterior mean for squared error loss, hold.

REMARK 5.1. (1) General set of conditions of posterior convergence through the LAN property was given in Ghosal et al. [11] extending methods in Ibragimov and Khasminskii [16]. For the model here, using the LAN property of the model along with Lemma 3.1-Lemma 3.3 in Huebner and Rozovskii [15], one verifies the conditions in Ghosal $e t$ al. [11] trivially and obtains the in probability version of the Bernstein-von Mises theorem and asymptotic equivalence in probability of the MLE and the Bayes estimators. However, we obtained the almost sure versions of these results.

(2) Large deviations and Berry-Esseen inequality for the MLE through the spectral approach were recently obtained by Bishwal et al. [4]. Extension of this problem to Bayes estimators remains to be investigated. Also to obtain rates of convergence of the posterior distributions to normal distribution and bounds on the asymptotic equivalence of the MLE and the Bayes estimators remains to be investigated.

(3) Sequential estimation in parabolic SPDEs using the spectral approach was studied by Bishwal and Sørensen [5].

(4) Nonparametric estimation of the coefficients of SPDEs is studied in Ibragimov and Khasminskii [17].

\section{Acknowledgements}

The author would like to thank the anonymous referee for careful reading of the paper and for suggestions which improved the presentation.

\section{References}

[1] I. V. Basawa and B. L. S. Prakasa Rao, Statistical inference for stochastic processes (Academic Press, New York, 1980).

[2] J. P. N. Bishwal, 'Bayes and sequential estimation in Hilbert space valued stochastic differential equations', J. Korean Statist. Society 28 (1999), 93-106.

[3] _ 'Rates of convergence of the posterior distributions and the Bayes estimators in the OrnsteinUhlenbeck process', Random Operators and Stochastic Equations 8 (2000), 51-70.

[4] J. P. N. Bishwal, B. Markussen and M. Sørensen, 'Large deviations and Berry-Esseen inequality for the maximum likelihood estimator in parabolic SPDEs', preprint, 2000.

[5] J. P. N. Bishwal and M. Sørensen, 'Sequential estimation in parabolic SPDEs', preprint, 2000.

[6] J. D. Borwanker, G. Kallianpur and B. L. S. Prakasa Rao, 'The Bernstein-von Mises theorem for Markov processes', Ann. Math. Statist. 42 (1971), 1241-1253. 
[7] A. Bose, 'The Bernstein-von Mises theorem for a certain class of diffusion processes', Sankhyā Ser. A 45 (1983), 150-160.

[8] L. Le Cam, 'On some asymptotic properties of maximum likelihood and related Bayes estimators', Univ. Calif., Publ. in Statist. 1 (1953), 227-330.

[9] L. Le Cam and G. L. Yang, Asymptotics in statistics: some basic concepts (Springer, New York, 1990).

[10] R. Carmona and B. L. Rozovskii, Stochastic partial differential equations: six perspectives (Amer. Math. Soc., Rhode Island, 1999).

[11] S. Ghosal, J. K. Ghosh and T. Samanta, 'On convergence of posterior distributions', Ann. Statist. 23 (1995), 2145-2152.

[12] H. Holden, B. Øksendal, J. Ubøe and T. Zhang, Stochastic partial differential equations (Birkhäuser, Boston, 1996).

[13] M. Huebner, 'A characterization of asymptotic behaviour of maximum likelihood estimators for stochastic PDEs', Math. Methods Statist. 6 (1997), 395-415.

[14] M. Huebner, R. Z. Has'minskii and B. L. Rozovskii, 'Two examples of parameter estimation for stochastic partial differential equations', in: Stochastic processes (eds. S. Cambanis, J. K. Ghosh, R. L. Karandikar and P. K. Sen), Festschrift in Honour of G. Kallianpur (Springer, Berlin, 1993) pp. 149-160.

[15] M. Huebner and B. L. Rozovskii, 'On the asymptotic properties of maximum likelihood estimators for parabolic stochastic PDEs', Probab. Theory Related Fields 103 (1995), 143-163.

[16] I. A. Ibragimov and R. Z. Khasminskii, Statistical estimation: asymptotic theory (Springer, Berlin, 1981).

[17] —-, 'Estimation problems for coefficients of stochastic partial differential equations. Part I', Theory Probab. Appl. 43 (1998), 370-387.

[18] K. Itô, Foundations of stochastic differential equations in infinite dimensional spaces, CBMS-NSF Regional Conference Series in Appl. Math. 49 (SIAM, Philadelphia, Pennsylvania, 1984).

[19] G. Kallianpur and X. Xiong, Infinite dimensional stochastic differential equations, Lecture NotesMonograph Series 26 (Institute of Mathematical Statistics, Hayward, California, 1995).

[20] T. Koski and W. Loges, 'Asymptotic statistical inference for a stochastic heat flow problem', Statist. Prob. Letters 3 (1985), 185-189.

[21] __ 'On minimum contrast estimation for Hilbert space valued stochastic differential equations', Stochastics 16 (1986), 217-225.

[22] Yu. A. Kutoyants, Parameter estimation for stochastic processes, (Translated and edited by B. L. S. Prakasa Rao) (Heldermann, Berlin, 1984).

[23] _ Identification of dynamical systems with small noise (Kluwer, Dordrecht, 1994).

[24] _ Parameter estimation for ergodic diffusion processes, unpublished monograph, 1999.

[25] R. S. Liptser and A. N. Shiryayev, Statistics of random processes I, II (Springer, Berlin, 1977, 1978).

[26] W. Loges, 'Girsanov's theorem in Hilbert space and an application to the statistics of Hilbert space valued stochastic differential equations', Stochastic Process Appl. 17 (1984), 243-263.

[27] S. V. Lototsky and B. L. Rozovskii, 'Spectral asymptotics of some functionals arising in statistical inference for SPDEs', Stochastic Process Appl. 79 (1999), 69-94.

[28] M. N. Mishra, 'The Bernstein-von Mises theorem for a class of non-homogeneous diffusion processes', Statist. Decisions 7 (1989), 153-165.

[29] L. Piterbarg and B. L. Rozovskii, 'Maximum likelihood estimators in the equations of physical oceanography', in: Stochastic modelling in physical oceanography (eds. R. Adler, P. Muller and B. Rozovskii) (Birkhäuser, Boston, 1996) pp. 397-421.

[30] — - 'On asymptotic problems of parameter estimation in stochastic PDEs: discrete time sampling', Math. Methods Statist. 6 (1996), 200-223. 
[31] B. L. S. Prakasa Rao, 'The Bernstein-von Mises theorem for a class of diffusion processes', Theory Random Processes 9 (1980), 95-101 (in Russian).

[32] - 'On Bayes estimation for diffusion fields', in: Statistics: applications and new directions, Proc. ISI Golden Jubilee Conferences (eds. J. K. Ghosh and J. Roy) (Statist. Publ. Soc., Calcutta, 1984) pp. 504-511.

[33] - Statistical inference for diffusion type processes (Arnold, London, 1999).

[34] J. B. Walsh, An introduction to stochastic partial differential equations, Lecture Notes in Math. 1180 (Springer, Berlin, 1986) pp. 265-439.

Department of Economics

Fisher Hall

Princeton University

Princeton, NJ 08544-1021

USA

e-mail: jbishwal@princeton.edu 